\title{
The 100th Anniversary of the Norwegian Mathematical Society and the Viggo Brun Prize
}

Petter Andreas Bergh, President of the Norwegian Mathematical Society

The Norwegian Mathematical Society was founded in Oslo on 2 November 1918. During the first decades after 1900 , the mathematics community in Norway grew substantially and the society was established with the intention of fostering contact between the nation's mathematicians. The society is amongst the smaller ones in Europe, with a little over 300 members as of 2018. It serves as the main professional organisation for mathematicians in Norway and works to promote research, cooperation and recruitment. Its monthly newsletter, Infomat, is freely available online.

The society's activities are aimed at mathematicians in all stages of their careers, as illustrated by the follow- ing three examples. Every year, it organises a national competition for high school students, the Abel Competition, which also serves as the national qualification for the International Mathematical Olympiad. This is a very popular competition and the winners usually receive their awards from the minister of education at a prize ceremony. The society also awards Abel Scholarship Grants. These are grants awarded annually to some of the best students on Master's programmes in Norway, supporting shorter and longer visits to universities abroad. Finally, each year, the society organises the Abel Symposium, a conference with distinguished international speakers. These three activities are funded by the Abel Prize. 


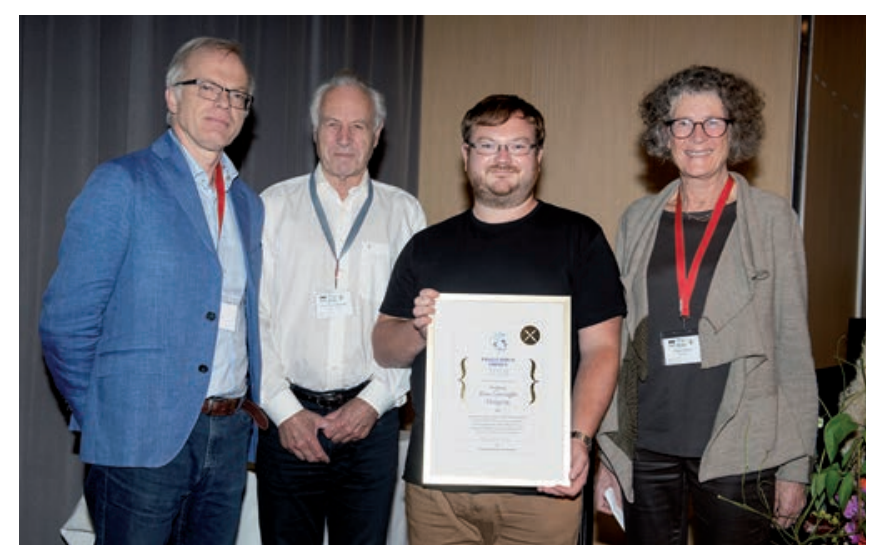

From left to right: Kristian Seip, Finn Faye Knudsen, Rune Haugseng (recipient of the Viggo Brun Prize) and Ragni Piene. The photo is courtesy of Helge Skodvin.

In September 2018, the society celebrated its 100th anniversary at a national meeting of mathematicians held in Bergen. At the meeting, the society announced a new national prize, the Viggo Brun Prize (see https://web. matematikkforeningen.no/viggo-brun-prize/):
The prize is named after the noted number theorist Viggo Brun, who became an honorary member of the Norwegian Mathematical Society in 1974. It is to be awarded biannually to a young mathematician, and comes with a cash reward of NOK 50.000. The 2018 laureate is Rune Haugseng, who receives the prize for his fundamental contributions to the theory of higher categories, with applications to quantum field theory, representation theory, algebraic geometry, and geometric topology, and for the development of higher Morita theory and enriched $\infty$-categories.

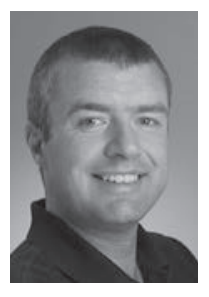

Petter Andreas Bergh is a professor of mathematics at the Norwegian University of Science and Technology (NTNU) in Trondheim, Norway. His research interests are in homological algebra, triangulated categories and representation theory of algebras. He is currently the President of the Norwegian Mathematical Society. 